\title{
Towards a Sensor-based System for Assessing and Monitoring Powered Mobility Skills in Children
}

\author{
Roxana Ramirez Herrera ${ }^{1}$, Catherine Holloway ${ }^{1}$, Behzad Momahed Heravi ${ }^{1}$, Tom Carlson ${ }^{2}$ \\ ${ }^{1}$ UCL Interaction Centre \\ University College London, \\ Gower Street, London, WC1E 6EA, United Kingdom \\ ${ }^{2}$ Aspire CREATe Centre for Rehabilitation \\ Engineering and Assistive Technology \\ University College London, \\ \{roxana.herrera.15, c.holloway, b.heravi\}@ucl.ac.uk \\ Stanmore, HA7 4LP, United Kingdom \\ t.carlson@ucl.ac.uk
}

\begin{abstract}
Children with motor or cognitive impairments who require powered mobility at a very young age will face social and environmental barriers that make learning how to use the mobility device a challenging task. We present a first approach of a framework to help therapists and service providers to assess and monitor how children use their mobility device, which results from the combination of a plug and play inertial sensor, and the support of the Assessment Learning tool (ALP) from Nilsson and Durkin. We performed a formative study on four able-bodied children using an electric wheelchair. Results suggest it is possible to measure children's driving skills with this approach, and that results can be mapped to the validated ALP tool. We present the limitations of our study and the direction of future work.
\end{abstract}

\section{Keywords}

Powered mobility; assessment and monitoring; children; wheelchair skills; IMU.

\section{INTRODUCTION}

During infancy, independent movement enables children to interact with the world and this exploration contributes to their social, emotional, cognitive, visual and perceptual development [2][4]. In children with certain motor impairments, the lack of independent mobility within their first years of life can severely limit this interaction, compromising their development and contributing to passivity and dependence [4]. The provision of powered mobility devices (such as electric wheelchairs) has shown to compensate for the potential development issues posed by immobility [5]. These benefits however, are diminished without adequate training and practice, particularly for those children with cognitive or sensory limitations which are in greater need of guided personalized support [5]. An effective personalized training therapy depends not only on therapy sessions but also on the correct assessment and follow-up of the child's driving skills at each of its developmental stages[5]. Yet, public healthcare systems often find themselves unable to provide enough or even any type of qualified or personalized clinical support in the long term [2]. In consequence, some children's development will largely depend on when and how much they practice powered mobility at their everyday setting, such as home or school. Hence, we propose a sensor-based system for assessing

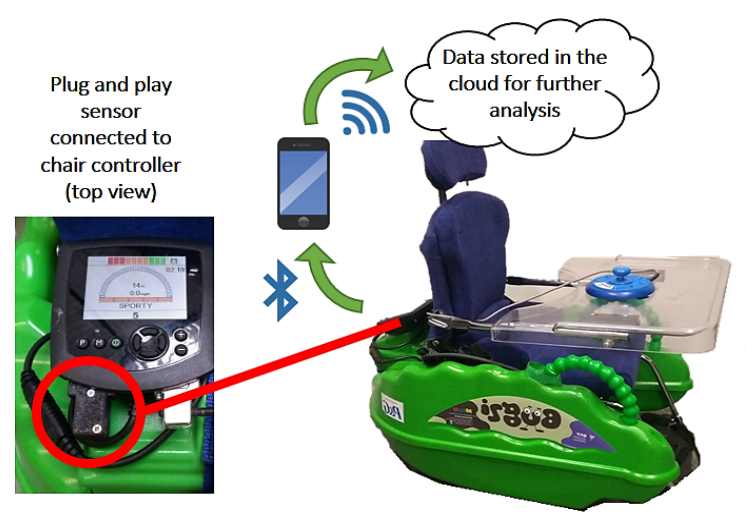

Figure 1. Diagram of proposed framework.

and monitoring powered mobility skills in children in their everyday settings (see Figure 1).

The system would need to objectively measure the level of driving skills of children and be validated against the current assessment method performed by healthcare practitioners. As a first step, we present our framework and a formative study in which we use an inertial measurement unit (IMU) to measure the child's driving learning stage.

\section{RELATED WORK}

The Assessment to Learn Powered mobility use (ALP) in early childhood is a fairly recent qualitative tool designed by Durkin and Nilsson that has been successfully used and appraised in the UK and Sweden [6]. It identifies eight ascending stages in the process of learning powered mobility use: novice, curious novice, beginner, advanced beginner, sophisticated beginner, competent, proficient and expert. Each stage description provides extensive cues for the therapist in five different observational categories: "level of attention," "activity and movement," "understanding of tool use," "expressions and emotions," and "interaction and communication" [6]. Like the ALP, existing assessment tools used for driving skills are typically subject to the therapist interpretation.

Wheelchair usage in particular has been characterized by a range of sensors to objectively measure driving behavior within both the users' daily environments and controlled settings for rehabilitation or evaluation [7]. Inertial sensors (IMUs) are the most recurrent sensor type, and their wide use can be attributed to the simplicity of installation and recently decreasing price in the market [3]. Most of these studies however, have been performed with adult users and some with able-bodied subjects, while only a few with children; the latter studies taking place mostly in controlled environments [4]. These studies have used existing assessment tools, or have proposed their own evaluation parameters, but have not used yet the ALP scale. To our knowledge, no studies have 
been performed on children under the age of 5 , in their everyday settings.

\section{CONCEPT PROPOSAL}

Our framework (see Figure 1) proposes the use of a plug and play sensor (an IMU) that will be distributed to the legal guardians of toddlers that use powered mobility. This plug and play device will be powered by the electric wheelchair, making it easy to use and reducing the barrier to taking part in the study.

The child will use the wheelchair within their everyday settings (for example, school, home or therapy center), and relevant parameters for their skills characterization will be recorded. This plug and play device will automatically transfer, via Bluetooth, pre-recorded data to a smart phone at regular intervals (for example, once every two days). Data will then be transferred via $\mathrm{Wi}-\mathrm{Fi}$ to the Cloud, in which it will be processed, analyzed and distributed to researchers, therapists and electric wheelchair providers.

The first stage of the framework development will be to extract information from current users that have already been assessed using the ALP tool and classified to one of the 8 learning stages. Finding enough children at each stage of development would be challenging in a local area. However, using this platform could enable us to surpass geographical constrains and collect and record power assistive usage of children on a larger scale and simultaneously. During the processing stage, usage patterns can be extracted which can be used to create a database of average parameters for each of the stages. This can work as a guiding principle for classifying users at the second stage of the framework. Data analysis can also highlight the potential difficulties that the user might be experiencing while using the wheelchair when compared to a baseline.

The final system will include a database that allows remote assessment of powered mobility skills to help whenever needed. due to restrictions on time or location, access to therapist's live evaluation is difficult if not impossible. The system can assess the user and guide the parents, caregivers or therapist on strategies for intervention when appropriate. The baseline will be constantly updated as users join the network.

\section{FORMATIVE STUDY}

We performed a formative study to explore whether an IMU plug and play prototype could be used to describe the learning stage of the child from the ALP observational category "activity and movement". We recruited four able-bodied children between 4 and 5 years old, and asked them to perform a set of 12 tasks within a rectangular area, using the Bugzi[1] (Figure 1), an indoor powered wheelchair for toddlers, controlled with a joystick. The tasks involved moving with the chair in a straight line between two points, following rectangular or triangular trajectories, and a free trajectory period. Each session was video recorded, and triaxial acceleration and angular rate of the Bugzi were measured with the IMU sensor (accelerometer and gyroscope). These signals were filtered and plotted for each child in MATLAB. From these plots, we identified 4 types of movements: idle, rotation to the left, rotation to the right, and straight line. Each child's skill level was classified using the ALP through a mixture of researcher observations both in person and on re-watching the video. From these observations and IMU data, we figured that the time each child spent performing different types of movement could be indicative of their skills level. The time spent on each type of activity was calculated by an algorithm which classified the types of movements by comparing both acceleration and angular rate against thresholds which were empirically deduced form observing several plots. Data suggests (see Table 1) that a higher amount of rotations (either left or right on the chair) represents constant correction of the trajectory and maps to the child who scored the lowest on the ALP tool. On the other hand, the child who scored the highest, performed confidently on the use of the wheelchair and executed less rotations when performing tasks. The other participants did not differ significantly on straight movement performance. These findings are congruent with Nilsson's and Durkin's observational descriptions.

Table 1. ALP classification and percentage of time per activity

\begin{tabular}{|c|c|c|c|}
\hline Child & ALP stage & \%time rotating & \%time straight line \\
\hline 1 & expert & 17.6 & 57.3 \\
\hline 2 & proficient & 23.5 & 52.8 \\
\hline 3 & proficient & 35.4 & 56.1 \\
\hline 4 & competent & 40.7 & 30.3 \\
\hline
\end{tabular}

\section{CONCLUSION AND FUTURE WORK}

As a first step to developing the sensing component of our framework, we propose the use of an IMU plug and play prototype. The data from our formative study suggests a relation between the level of competence of Bugzi driving, and the amount of time that the children spent rotating the chair, which is probably due to trajectory correction. Future work will improve the experimental design to better deal with the challenges of working with children, such as dealing with their playfulness with the mounted camera and sensor. Since the tool is created to look at functional output rather than disability, we believe these results are useful indicator of what would have been captured with disabled children. However, this needs to be validated. As our results are preliminary and our sample group is not representative, we will then proceed with the collection of more data from a larger number of participants both able-bodied and motor impaired in their everyday settings. Lastly, we will proceed to use a more efficient and automatic algorithm for movement type classification, by taking advantage of the many machine learning tools available.

\section{ACKNOWLEDGEMENTS}

This project was funded by the Queen Elizabeth's Foundation for disabled people, and by CONACyT.

\section{REFERENCES}

[1] Bugzi - MERU: http://meru.org.uk/what-we-do/bugzi/.

[2] Feldner, H.A. et al. 2015. Why the time is right for a radical paradigm shift in early powered mobility: the role of powered mobility technology devices, policy and stakeholders role of powered mobility technology devices, policy and stakeholders. Disabil Rehabil Assist Technol. early onli, April (2015).

[3] Fu, J. et al. 2016. A novel mobile-cloud system for capturing and analyzing wheelchair maneuvering data: A pilot study. Assist Technol. 28, 2 (2016), 105-114.

[4] Livingstone, R. and Field, D. 2014. Systematic review of power mobility outcomes for infants, children and adolescents with mobility limitations. Clin Rehabil. 28, 10 (2014), 954-964.

[5] Livingstone, R. and Paleg, G. 2014. Practice considerations for the introduction and use of power mobility for children. Dev Med Child Neurol. 56, 3 (2014), 210-221. 
[6] Nilsson, L. and Durkin, J. 2014. Assessment of learning powered mobility use--applying grounded theory to occupational performance. J Rehabil Res Dev. 51, 6 (2014), 963-74.

[7] Routhier, F. et al. 2016. Data Logger Technologies for Manual Wheelchairs: A Scoping Review. Assist Technol. 00, 00 (2016), 1-8. 